\title{
Resistance scaling at the Kosterlitz-Thouless transition
}

\author{
Mats Wallin ${ }^{1}$ and Hans Weber ${ }^{2}$ \\ ${ }^{1}$ Department of Theoretical Physics, Royal Institute of Technology, S-100 44 Stockholm, Sweden \\ ${ }^{2}$ Department of Physics, Luleå University of Technology, S-971 87 Luleå, Sweden.
}

(August 24, 2017)

\begin{abstract}
We study the linear resistance at the Kosterlitz-Thouless transition by Monte Carlo simulation of vortex dynamics. Finite size scaling analysis of our data show excellent agreement with scaling properties of the Kosterlitz-Thouless transition. We also compare our results for the linear resistance with experiments. By adjusting the vortex chemical potential to an optimum value, the resistance at temperatures above the transition temperature agrees well with experiments over many decades.
\end{abstract}

PACS numbers: 05.70Jk, 64.60Cn, 74.20De 
Scaling of current-voltage characteristics is useful both in theoretical and experimental analysis of superconductors. One useful way to calculate linear and nonlinear resistance accurately is by computer simulation, and scaling analysis is crucial to extract the critical properties. The linear resistance and nonlinear current-voltage characteristic are key quantities in the recently much studied vortex glass state in disordered superconductors [1] . At the vortex glass transition the nonlinear current-voltage characteristic is universal, and the linear resistance vanishes, signaling true superconductivity. The current-voltage characteristic is also a key quantity of the zero-field superconducting transition in two dimensions, which is a Kosterlitz-Thouless (KT) transition [2,3]. The resistance curve above the KT transition is given by an (unknown) universal function of a reduced Coulomb gas temperature variable, allowing experimental data for different 2D systems to collapse on the same curve, as shown by Minnhagen [⿴囗十 Remarkably, also certain 3D high temperature superconductors, with weakly coupled layers, seem to fit the same curve, except very close to $T_{c}$ [5, 6]. The term "universality" for the resistance curve is used in the same sense as e.g. the universality of the BCS gap function, rather than in the sense of critical phenomena. The form of the resistance curve has been accurately constructed from such experiments, but an accurate calculation is lacking. KT theory gives an approximate expression for the resistance, valid in the KT critical region, but which cannot accurately fit the experimental curve outside the KT critical region (this will be seen below). One calculation going beyond the approximate KT resistance expression was a simulation of the sine-Gordon formulation of the Coulomb gas [7]. An indirect connection was found to experiments, but a direct calculation of the resistance curve is lacking.

In this paper we report results of Monte Carlo (MC) simulation and finite size scaling analysis of the linear resistance, from equilibrium vortex dynamics of the 2D Coulomb gas model for the KT transition. We first use finite size scaling analysis to extract critical properties from our MC data for the linear resistance, and we find excellent agreement with scaling properties of the KT transition. Secondly, we show that under certain conditions, our results for the linear resistance can closely fit the experimental curve above the transition temperature.

Let us first consider scaling properties of the linear resistance at a superconducting transition driven by vortex fluctuations, like the KT transition or the vortex glass transition. At the critical temperature $T_{c}$ the correlation length diverges: $\xi \rightarrow \infty$. Dynamic quantities like the resistance depend on the correlation time $\tau$, which diverges as $\tau \sim \xi^{z}$, where $z$ is the dynamical exponent. Voltage is related to the rate of phase slip of the superconducting order parameter, $V=\hbar /(2 e) \dot{\phi} \sim \xi^{-z}$. The linear resistance is related to the equilibrium voltage fluctuations according to the Nyquist formula [8 [10]

$$
R=\frac{1}{2 T} \int_{-\infty}^{+\infty} d t\langle V(t) V(0)\rangle
$$

and thus the resistance scales as $R \sim \xi^{-z}$.

Now we specialize to two dimensions. The superconducting transition is now a KT transition, where confined neutral vortex pairs start to deconfine, so that "free" vortices appear. Here we will assume the value $z=2$ [11,12], which describes free vortex diffusion. Hence the resistance is expected to scale like

$$
R \sim \xi^{-2} .
$$


This scaling form, here obtained from general scaling arguments, is the same as from the usual KT theory (see below). Simulations are done in finite systems, and the diverging correlation length at $T_{c}$ is cut off at the system size $L$. Precisely at $T_{c}$ the resistance is therefore expected to obey $R \sim L^{-2}$, or $L^{2} R=A$, where $A$ is a (nonuniversal) constant.

As an aside comment, we note that another way of introducing a finite length scale at the transition is to apply a finite current density $J$. The finite current length $\xi_{J}$, defined by $\xi_{J} J=k_{B} T$ [1], is the length beyond which the diverging correlations at the transition are driven out of equilibrium by the Lorentz force. The resulting nonlinear response obeys $E=R(J) J \sim \xi_{J}^{-z} J \sim J^{z+1}=J^{3}$, which is the usual KT result, here obtained within a general scaling approach.

We can extract the leading finite size correction to scaling of the resistance by using known results from KT theory. The correlation length $\xi$ diverges as [4]

$$
\xi^{-2} \sim\left[g \frac{4 \pi z}{\epsilon T}\right]^{1 /\left(1-\frac{1}{4 \epsilon T}\right)},
$$

for $T \rightarrow T_{c}^{+}$, where $g$ is a constant, $1 / \epsilon$ is a dielectric function proportional to the renormalized superfluid density, and $z$ is the fugacity. The Bardeen-Stephen relation [13], $R / R_{N}=2 \pi \zeta^{2} n_{F}$, ties the resistance to the density of free vortices $n_{F}$, where $R_{N}$ is the normal state resistance and $\zeta$ the Ginsburg-Landau length, and $n_{F}$ is in turn related to $\xi$ according to the Debye-Hückel relation [4], $\xi^{-2}=2 \pi n_{F} / \epsilon T$. Eliminating $n_{F}$ gives back the scaling relation, $R \sim \xi^{-2}$, in agreement with Eq. (21). The leading correction to scaling is known for $1 / \epsilon$ [14]: $1 / \epsilon(L)=1 / \epsilon(\infty)(1+1 /(2 \ln (L)+C))$. Combining these relations gives again a finite size scaling relation for $R$, similar to $L^{2} R=A$, but now containing the leading logarithmic correction:

$$
L^{2} R\left(1+\frac{1}{4 \ln (L)+C}\right)=A,
$$

where $C$ is an unknown constant. We also need the standard $\mathrm{KT}$ form of the resistance [1]. Using the temperature dependence of $\epsilon$ from KT theory, $\xi$ becomes $\xi \sim \exp \left(\right.$ const $\left./ \sqrt{T-T_{c}}\right)$, which gives

$$
R \sim e^{-\mathrm{const} / \sqrt{T-T_{c}}},
$$

expected to be valid in the KT critical scaling region.

In the simulation we use the $2 \mathrm{D}$ Coulomb gas model. We consider a square lattice with $N=L^{2}$ sites with periodic boundary conditions. The grand partition function is $Z=\operatorname{Tr}_{\left\{n_{j}\right\}} \exp (-H / T)$, with

$$
H=\frac{1}{2} \sum_{i, j} n_{i} n_{j} G_{i j}-\mu \sum_{j} n_{j}^{2},
$$

where the trace is over $n_{i}=0, \pm 1$, where $n_{i}=0$ means no vortex and $n_{i}= \pm 1$ means one vortex of vorticity \pm at site $i$. The parameter $\mu=-E_{c}$ is a "vortex chemical potential" and $E_{c}$ is the vortex core energy. We only allow configurations of zero total vorticity. The vortex-vortex interaction is a lattice Green's function, $G(\mathbf{r})=(2 \pi / N) \sum_{\mathbf{k} \neq 0} \exp (i \mathbf{k} \cdot \mathbf{r}) /(4-$ 
$2 \cos k_{x}-2 \cos k_{y}$ ), which varies like $-\ln r$ on large distances (the lattice spacing is set to $a=1)$.

Now we will describe the simulation. Following Ref. [12], our MC algorithm consists of attempts to insert near neighbor $(n=+1, n=-1)$ pairs, on randomly chosen lattice positions, and with random orientation, and we test for acceptance according to the Metropolis algorithm. We typically discard $10^{4}$ initial sweeps through the system to approach equilibrium, and then take measurements during up to $10^{6}$ sweeps close to $T_{c}$, and fewer away from $T_{c}$. We compute the linear resistance in the simulation by evaluating the Nyquist formula, Eq. (11), as a sum over discrete time steps [9:10]. The voltage $V(t)$ is given by \pm 1 (in suitable units), if at MC time $t$ insertion of a vortex pair with orientation $+-(-+)$ is accepted, and $V(t)=0$ otherwise. To equate $\mathrm{MC}$ time to real time assumes heavily overdamped dynamics, which should be fulfilled near the transition.

Now we turn to the results. In Figure 1 we determine the transition temperature from finite size scaling of MC data for the linear resistance. The vortex chemical potential was here chosen to $\mu=0$, which gives the Coulomb gas corresponding to the 2D XY model in the Villain approximation. According to Eq. (2) data for $L^{2} R$ for different lattice sizes $L$ should be independent of system size at $T=T_{c}$. This means that $T_{c}$ is where the curves in the figure cross. Note that this scaling procedure assumes initial knowledge of the dynamical exponent, $z=2$. Fig. 1 (a) is based on Eq. (2) and does not contain corrections to scaling. Fig. 1 (b) is based on Eq. (四) which contains the lowest logarithmic correction to scaling. The constant $C$ in Eq. (田) was adjusted to as closely as possible have all curves cross at a single point. Comparing (a) and (b) shows that including the correction significantly improves the fit, and gives the estimate $T_{c} \approx 0.218$ for $\mu=0$. Error bars on the data points have been left out because they are much smaller than the symbol size.

Figures 1 (a) and (b) show that the resistance obeys the expected scaling form $R \sim L^{-2}$ at the transition, $T=T_{c}$. This also means that in the superconducting phase below $T_{c}$, the resistance scales to zero with system size. This is possible in the Nyquist formula (四), despite that the equal-time voltage correlation, $\left\langle V^{2}(0)\right\rangle$, gives a finite, roughly size independent, contribution to the resistance at all finite temperatures, also below $T_{c}$. What happens is that below $T_{c}$ the unequal-time correlations on average cancel the equal-time correlations. This cancellation is incomplete above $T_{c}$, and in finite systems also below. Within the KT vortex unbinding picture, below $T_{c}$ all vortices are bound in neutral pairs. Creation of a pair gives a burst of voltage that contributes to the equal-time voltage correlation, but if the pair is later annihilated a canceling unequal-time correlation results. Above $T_{c}$ some pairs unbind, the cancellation becomes incomplete, and the resistance is finite.

We wish to compare the present approach of using the linear resistance to locate the critical point, to the often used method of locating the universal jump in the superfluid density $\rho_{s}$. In the Coulomb gas $\rho_{s}$ corresponds to the dielectric response function $\rho_{s} \sim$ $1 / \epsilon(k \rightarrow 0)$, where $1 / \epsilon(\mathbf{k})=1-2 \pi /\left(k^{2} T N\right)\left\langle|n(\mathbf{k})|^{2}\right\rangle$, where $n(\mathbf{k})$ the the Fourier transform of the vortex density. The strong size dependence of $R \sim L^{2}$ produces much stronger splay of data away from $T_{c}$, than the universal jump which has size dependence $\rho_{s} \sim L^{0}$. This makes locating $T_{c}$ easier. Furthermore, use of $1 / \epsilon(\mathbf{k})$ is in practice limited to the smallest available nonzero wave vector, $k=2 \pi / L$, while $R$ is for $k=0$.

Now we will compare resistance data to the experimental curve over a finite temperature interval above $T_{c}$. In order to do this we first employ a crucial empirical correction to our 
$\mathrm{MC}$ results. Our discrete MC dynamics fails in the limit of high temperature, because the voltage fluctuations saturate at high $T$ when most MC moves are accepted, which incorrectly makes the resistance vanish like $R \sim 1 / T \rightarrow 0$ according to Eq. (1). Instead of approaching a constant at high $T$, we assume an additional factor of $T$ in the vortex velocity squared, as for the temperature dependence of a diffusion process according to the Einstein relation [8]. This correction cancels the factor $1 / T$ in the Nyquist formula, and makes $R$ approach a constant at high $T$. The correction is negligible in the critical region, compared to the strong exponential temperature dependence in Eq. (5).

Figure 2 (a) shows MC data for the resistance curve, together with the experimental curve. Note that the temperature scale is here the Coulomb gas temperature, $T=T^{\mathrm{CG}}$, which differs from the physical temperature by the temperature dependence of the unrenor-

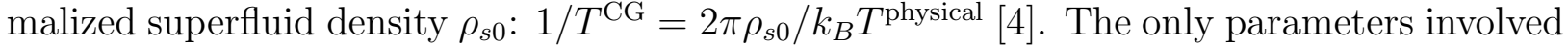
in the plot are $T_{c}$, which is determined separately by the above scaling approach, and the normalization constant $R_{N}$, which is adjusted to obtain agreement with the experimental curve at high $T$. MC data for $\mu=0$ is seen to deviate strongly from the experimental curve. To get better agreement we tried different values of the vortex chemical potential $\mu$. We obtain best agreement for the value $\mu=-0.8$. The critical temperature for $\mu=-0.8$ is very close to the upper limit $T_{c}=1 / 4$, and the value $T_{c}=1 / 4$ is used in the plot. Further decreasing $\mu$ gives a worse fit. Figure 2 (b) is a KT plot with $1 /\left(T / T_{c}-1\right)^{-1 / 2}$ on the x-axis. The KT form given by Eq. (5) corresponds to a straight line in this plot.

Let us discuss this result. By adjusting the vortex chemical potential to $\mu=-0.8$, our data for the resistance is in close agreement with the experimental curve over many decades, and over a broad temperature range. The agreement is not perfect, as indicated by the finite size effects near $T_{c}$ in Fig. 2, but the agreement is clearly better than a fit to KT theory outside the KT critical region. The value $\mu=-0.8$ seems perhaps unexpectedly small, compared to e.g. the $2 \mathrm{D} X Y$ model which has $\mu \approx 0$. The corresponding Coulomb gas model has very small vortex density, and it is very close to the KT line of fixed points. One possible interpretation is that this is actually an effective vortex model with renormalized parameters. For example, vortex crystallization in superconducting films was suggested recently by Gabay and Kapitulnik [15]. Our Coulomb gas model could describe logarithmically interacting vacancies with big core energy.

We have also studied the effect of adding random vortex pinning, modeled as a random site energy for the vortices. This shifts $T_{c}$ downwards, but gives exactly the same scaling properties at the new $T_{c}$. This directly verifies the expectation that weak disorder is indeed irrelevant at the KT transition, in agreement with the Harris criterion, $\nu>2 / d$ [16,17.

In summary, our results from finite size scaling of $\mathrm{MC}$ data for the linear resistance of the 2D Coulomb gas are in excellent agreement with scaling properties of the KT transition. This shows that Monte Carlo dynamics is in the right dynamical universality class, and allows accurate determination of the critical temperature. Furthermore, by tuning the vortex chemical potential, our results can closely reproduce the experimental universal resistance curve over many decades, and the agreement outside the KT critical region is much better than a simple fit to the KT theory.

We acknowledge stimulating discussions with S. M. Girvin, P. Minnhagen, H. J. Jensen, and S. Teitel. This work was supported by grants from the Swedish Natural Science Research Council (NFR). 


\section{REFERENCES}

[1] M. P. A. Fisher, Phys. Rev. Lett. 62, 1415 (1989); D. S. Fisher, M. P. A. Fisher, and D. A. Huse, Phys. Rev. B 43, 130 (1991).

[2] J. M. Kosterlitz and D. J. Thouless, J. Phys. C 5, L124 (1972); 6, 1181 (1973).

[3] V. L. Berezinskii, Zh. Eksp. Teor. Fiz. 61, 1144 (1971) [Sov. Phys. JETP 34, 610 (1972)].

[4] For a review see: P. Minnhagen, Rev. Mod. Phys. 59, 1001 (1987).

[5] P. Minnhagen, Solid State Comm. 71, 25 (1989); P. Minnhagen and P. Olsson, Proc. Nobel Jubilee Symposium 1991, Phys. Scripta Vol. T42, 29 (1992); P. Minnhagen and P. Olsson, Phys. Rev. B 45, 5722 (1992).

[6] S. Martin, A. T. Fiory, R. M. Flemming, G. P. Espinosa, and A. S. Cooper, Phys. Rev. Lett. 62, 677 (1989); Phys. Rev. Lett. 67, 1039 (1991).

[7] P. Minnhagen and H. Weber, Phys. Rev. B 32, 3337 (1985); H. Weber and P. Minnhagen, Phys. Rev. B 38, 8730 (1988).

[8] F. Reif, Fundamentals of statistical and thermal physics, McGraw-Hill (1965).

[9] A. P. Young, in Proceedings of the Ray Orbach Inauguration Symposium, World Scientific (1993).

[10] R. A. Hyman, M. Wallin, M. P. A. Fisher, S. M. Girvin, and A. P. Young, unpublished.

[11] V. Ambegoakar, B. I. Halperin, D. R. Nelson, and E. D. Siggia, Phys. Rev. Lett. 40, 783 (1978); Phys. Rev. B 21, 1806 (1980).

[12] J. R. Lee and S. Teitel, Phys. Rev. Lett. 64, 1483 (1990); Phys. Rev. B 46, 3247 (1992); and unpublished.

[13] J. Bardeen and M.J. Stephen, Phys. Rev. 140, 1197 (1965).

[14] H. Weber and P. Minnhagen, Phys. Rev. B 37, 5986 (1988).

[15] M. Gabay and A. Kapitulnik, Phys. Rev. Lett. 71, 2138 (1993).

[16] A. B. Harris, J. Phys. C 7, 1671 (1974).

[17] M. Wallin, E. S. Sørensen, A. P. Young, and S. M. Girvin, Phys. Rev. B 49, 12115 (1994). 


\section{FIGURES}

FIG. 1. Location of the critical temperature by finite size scaling of Monte Carlo data for the linear resistance $R$. The critical temperature is located where data for different system sizes $L$ intersect. In (a) is shown data without the logarithmic correction to scaling, and in (b) is the same data including the logarithmic correction.

FIG. 2. Resistance curves from Monte Carlo simulations for different values of the vortex chemical potential $\mu$, together with the experimental curve. The resistance is plotted vs. $T$ in (a), and vs. $\left(T / T_{c}-1\right)^{-1 / 2}$ in (b) as suggested by Eq. (5). 